\title{
Combined MR direct thrombus imaging and non-contrast magnetic resonance venography reveal the evolution of deep vein thrombosis: a feasibility study
}

\author{
I. A. Mendichovszky ${ }^{1,2}$ - A. N. Priest ${ }^{1}$ - D. J. Bowden ${ }^{1} \cdot$ S. Hunter ${ }^{1} \cdot$ I. Joubert $^{1}$ \\ S. Hilborne ${ }^{1}$ - M. J. Graves ${ }^{1}$ T. Baglin ${ }^{3}$ - D. J. Lomas ${ }^{1,2}$
}

Received: 9 March 2016 / Revised: 9 June 2016 / Accepted: 9 August 2016/Published online: 30 August 2016

(C) The Author(s) 2016. This article is published with open access at Springerlink.com

\begin{abstract}
Objectives Lower limb deep venous thrombosis (DVT) is a common condition with high morbidity and mortality. The aim of the study was to investigate the temporal evolution of the acute thrombus by magnetic resonance imaging (MRI) and its relationship to venous recanalization in patients with recurrent DVTs.

Methods Thirteen patients with newly diagnosed lower limb DVTs underwent MRI with non-contrast MR venography (NC-MRV) and MR direct thrombus imaging (MR-DTI), an inversion-recovery water-selective fast gradient-echo acquisition. Imaging was performed within 7 days of the acute thrombotic event, then at 3 and 6 months.

Results By 3 months from the thrombotic event a third of the thrombi had resolved and by 6 months about half of the cases had resolved on the basis of vein recanalisation using NCMRV. On the initial MR-DTI acute thrombus was clearly depicted by hyperintense signal, while the remaining thrombi were predominantly low signal at 3 and 6 months. Some residual thrombi contained small and fragmented persisting hyperintense areas at 3 months, clearing almost completely by 6 months.
\end{abstract}

I. A. Mendichovszky and A. N. Priest are joint first authors

I. A. Mendichovszky

im391@cam.ac.uk

1 Department of Radiology, Addenbrooke's Hospital, Cambridge, UK

2 Department of Radiology, University of Cambridge, Cambridge, UK

3 Department of Haematology, Addenbrooke's Hospital, Cambridge, UK
Conclusions Our study suggests that synergistic venous assessment with combined NC-MRV and MR-DTI is able to distinguish acute venous thrombosis from the established (old) or evolving DVT detected by ultrasound.

Key Points

- MRI can distinguish between acute and evolving or chronic lower limb DVT

- Two advanced MRI techniques can follow the evolution of lower limb DVT

- MRI could be used to avoid an incorrect diagnosis of recurrent DVT

- MRI could help avoid the risks and complications of lifelong anticoagulation therapy

Keywords Magnetic resonance imaging · Venous thrombosis $\cdot$ Diagnosis $\cdot$ Longitudinal study $\cdot$ Clinical decision-making
Abbreviations
ADVANCE- Acceleration-dependent vascular anatomy for
MRV
CUS non-contrast-enhanced MR venography
DVT Compression ultrasonography
MR-DTI
Deep venous thrombosis
NC-MRV
Magnetic resonance direct thrombus imaging Non-contrast MR venography

\section{Introduction}

Lower limb deep venous thrombosis (DVT) is a common condition with high morbidity and mortality encountered both in community and hospital care [1]. Despite current anticoagulation-based treatments, the recurrence rate after a first episode of DVT remains high, ranging between $20 \%$ 
and $40 \%$ [2-4]. Current management of a recurrent DVT is lifelong anticoagulation therapy, bringing with it both the additional cost and the burden of monitoring, as well as the wellrecognized risks related to haemorrhage. These risks have to be balanced against the progression of a DVT with further complications such as pulmonary emboli that, in an important minority of cases, may be fatal [2, 4].

A clinically suspected DVT, often based on a positive Ddimer test and clinical scoring systems, is evaluated and followed up by compression ultrasound (CUS), now considered the standard diagnostic imaging test for lower limb DVT for the popliteal vein and above [5-8]. An acute symptomatic DVT is confirmed by CUS through the lack of compressibility of the common femoral, femoral and/or popliteal veins. However, the clinical presentation and diagnosis can be complicated by often ill-defined and occasionally absent symptoms that may accompany thrombus formation. In addition, many patients do not undergo follow-up imaging to confirm whether the affected vessels have fully or partially recanalised or remain occluded with chronic organized thrombus and increased collateral venous flow providing venous return.

Compression ultrasound has proven to be a simple, non-invasive, highly accurate, safe and cost-effective technique with high sensitivity and specificity of 89$100 \%$ and $87-100 \%$, respectively, for DVT of the popliteal vein and above in symptomatic patients [4, 9]. Despite all its advantages, CUS cannot reliably determine the age of a thrombus and thus distinguish an acute recurrent DVT from a persisting previous thrombus in the same location. Interpretation of CUS findings is further complicated by the persistence of US abnormalities after the initial episode $(80 \%$ of patients after 3 months and $50 \%$ of patients after 1 year from initial DVT diagnosis) $[4,7,10,11]$. Quantitative thrombus measurements have been proposed as a way to distinguish between new and recurrent DVT that, although with poor interobserver agreement in initial studies, have improved in recent publications [12, 13]. However, limited information remains available on the temporal evolution of an acute thrombus and our ability to distinguish between a resolving (old) or recurring (new) DVT.

Magnetic resonance direct thrombus imaging (MR-DTI) has been proposed as an alternative that may help establish thrombus age and distinguish between an acute recurrent thrombus and a persisting (evolving) clot in the same location [14-19]. Several studies have shown that MR-DTI is a highly accurate diagnostic test for an acute symptomatic DVT [14, 15]. The technique is based on the paramagnetic properties of methaemoglobin, formed in a fresh thrombus, which decreases the longitudinal relaxation time (T1), resulting in high signal on T1-weighted images. The MR-DTI highlights this bright thrombus signal by nulling the signals from fat (using a water-selective excitation) and blood (due to its long T1). The technique has been shown to have a high sensitivity and specificity for proximal DVT of the lower limbs (sensitivity 97$100 \%$; specificity $100 \%$ ) and good reproducibility [18-20].

Non-contrast MR venography (NC-MRV) has also been shown to be effective in demonstrating the peripheral venous system and diagnosing DVT in the thigh through visualization of filling defects within the venous lumen [21]. However, this technique alone cannot reliably distinguish between an acute and an evolving thrombus, as both of these entities will appear as intraluminal filling defects.

The aim of this study was to evaluate the temporal changes that occur in T1-weighted signal intensity and venous morphology in patients with a first acute DVT using combined MR-DTI and NC-MRV at three different time points after diagnosis. The understanding of the temporal evolution, as well as the accurate diagnosis of an acute thrombus, is clinically important, as it may help distinguish between a new clot (formed at or adjacent to the site of a previous DVT) and an evolving (old) thrombus, directly informing the clinical decision of lifelong anticoagulation in patients with recurrent DVT.

\section{Materials and methods}

\section{Patients}

Thirteen patients (mean age 61 years, range $18-78$ years; M/F $=11: 2$ ) with ultrasound-proven above-knee DVT (in the femoral vein and/or proximal popliteal vein) and no previous history of venous thrombosis or contraindications for MRI were recruited from the DVT service in our institution. The local ethics committee approved the study and all patients gave informed consent before undergoing MR imaging. The initial diagnosis of DVT was made on CUS by experienced sonographers as part of routine standard of care. Following patient recruitment, a member of the research team with ultrasound experience repeated the CUS study and the following parameters were recorded: the date/time of onset of symptoms, the patient's (pro-thrombotic) risk factors, the length of thrombus and its relationship to the inguinal ligament and knee joint space. CUS was also performed at 3 months (visit 2) and 6 months (visit 3) on the same day as the MRI followup. On these visits, CUS was only used to establish the presence of the thrombus.

MR imaging was performed on a 1.5-T scanner (Discovery MR450, GE Healthcare, Waukesha, WI) using a 12-channel phased array coil. The first examination (visit 1) was within 7 days of diagnosis, and further examinations were performed after 3 months (visit 2) and 6 months (visit 3). During this 6month period all the patients received standard anticoagulant therapy (warfarin) in accordance with local protocols. 


\section{MR examination}

For each examination, NC-MRV was acquired using Acceleration-Dependent Vascular Anatomy for Non-ContrastEnhanced MR Venography (ADVANCE-MRV) [22, 23]. This technique uses subtraction of velocity- and accelerationdependent angiography methods, as described previously [24, 25]. Dark-vein images are subtracted from bright-vein images to obtain vein-only images. The bright-vein images were acquired using an acceleration-sensitized acquisition to suppress the arterial blood, without suppressing venous blood [22, 23]. The dark-vein (velocity-sensitized) images were acquired using two consecutive velocity-sensitization modules with differing effective first gradient moments [22, 23]. To further ensure good venous suppression, four of these dark-vein image volumes were automatically acquired together and combined to give homogeneous venous signal $[22,23]$. The effective first gradient moments used to generate the dark-vein image volumes were 1.2, $0.6,0.3$ and $0.15 \mu \mathrm{Ts}^{2} / \mathrm{m}$. Additionally, a bright-artery image volume was acquired and used to generate an artery-only image volume by automated subtraction of the dark-artery (bright vein) images described above.

For these acquisitions, the image readout was a 3D balancedSSFP (oblique coronal orientation, flip angle $65^{\circ}$, echo time (TE) $1.7 \mathrm{~ms}$, repetition time (TR) $3.7 \mathrm{~ms}$, acquisition matrix $288 \times$ $288 \times 20$, reconstruction matrix $512 \times 512 \times 40$, field of view $(\mathrm{FoV}) 40 \times 40 \mathrm{~cm}$, slice thickness $2.4 \mathrm{~mm}$ ). The acquisition was cardiac gated using peripheral-pulse triggering with a trigger delay set to peak arterial flow. Parallel imaging (ASSET) was used with an acceleration factor of 2 . The acquisition time was 40 heartbeats per volume, or 240 heartbeats for one bright-vein, four dark-vein and one bright-artery image volumes. To avoid flow and heterogeneity-related artefacts near the edges of the field of view, two separate acquisitions covering the upper and lower halves of the thigh were performed, with a combined acquisition time of 480 heartbeats ( $8 \mathrm{~min}$ at $60 \mathrm{bpm}$ ). Based on the above matrix sizes, the acquired and reconstructed voxel dimensions were $1.38 \times 1.38 \times 2.4 \mathrm{~mm}^{3}$ and $0.78 \times 0.78 \times 1.2 \mathrm{~mm}^{3}$ respectively.

MR-DTI was performed using a 3D inversion-prepared fast gradient-echo acquisition with a water-selective excitation (1-2-1 binomial pulse sequence). The image volumes were acquired in coronal orientation and the image parameters were optimized by numerical simulation to ensure high signal from short-T1 thrombus while suppressing the signal from long-T1 blood [26]. The acquisition used centric k-space ordering, acquiring half the slice-encodes from each read-slice k-space plane per shot. The acquisition parameters were inversion time (TI) $340 \mathrm{~ms}$, TE $6.3 \mathrm{~ms}$, TR $12.2 \mathrm{~ms}$, flip angle $25^{\circ}$, centric ordering, acquisition matrix $320 \times 288 \times 96$, FoV $40 \times$ $40 \mathrm{~cm}^{2}$, slice thickness $2 \mathrm{~mm}, 320 \mathrm{~ms}$ delay after each shot. Parallel imaging (ASSET) was used with an acceleration factor of 2. Zero-filling interpolation was used in-plane to give a reconstructed volume size of $512 \times 512 \times 96$. The acquisition time was $5 \mathrm{~min} 59 \mathrm{~s}$.

\section{MR image analysis}

The MR-DTI volumes were reformatted to match the NCMRV images. An experienced radiologist (DJL) assessed the NC-MRV images using an Osirix workstation (Pixmeo, Berne, $\mathrm{CH}$ ) to determine the location and length of the venous occlusion (complete or partial, above-knee only). This assessment was performed principally using the subtracted NCMRV images; however, the arterial images, as well as the
Fig. 1 Images for an example patient showing the evolution of the thrombus signal. The NCMRV image (a composite MIP from the two table positions) shows an occluded right femoral vein at all three time-points, but the thrombus is visible on MRDTI only for the first examination. MR-DTI images are shown as curved-plane reformats
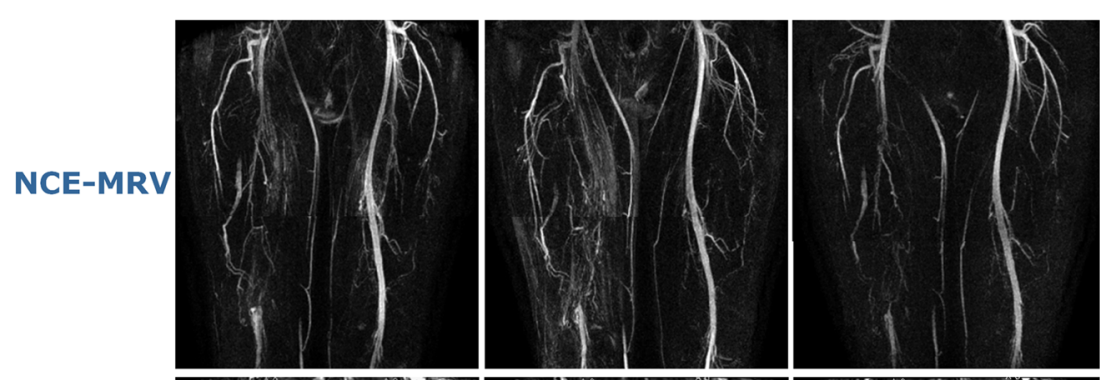

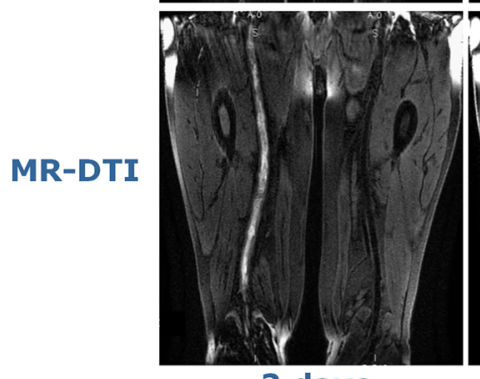

2 days

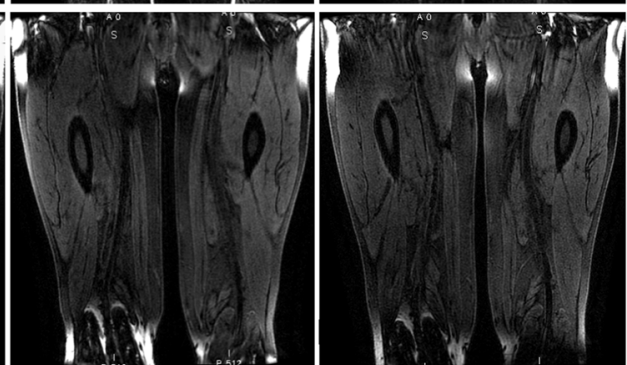

3 months
6 months 
Fig. 2 MIP images of the NCMRV showing an occluded right femoral vein at all three visits; curved-plane reformats of the MR-DTI show the whole thrombus at visit 1 , but only tiny fragments at visit 2 (arrows)

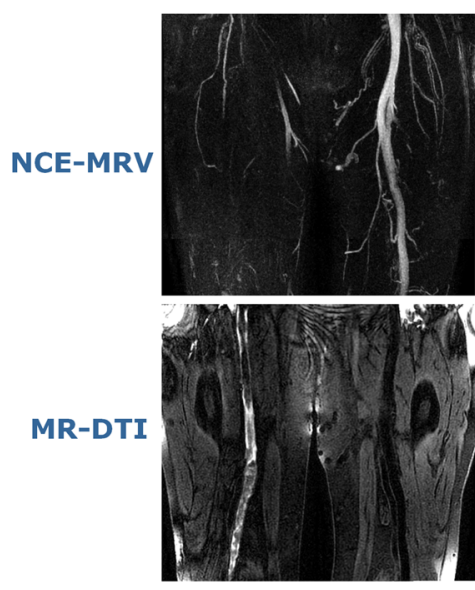

7 days
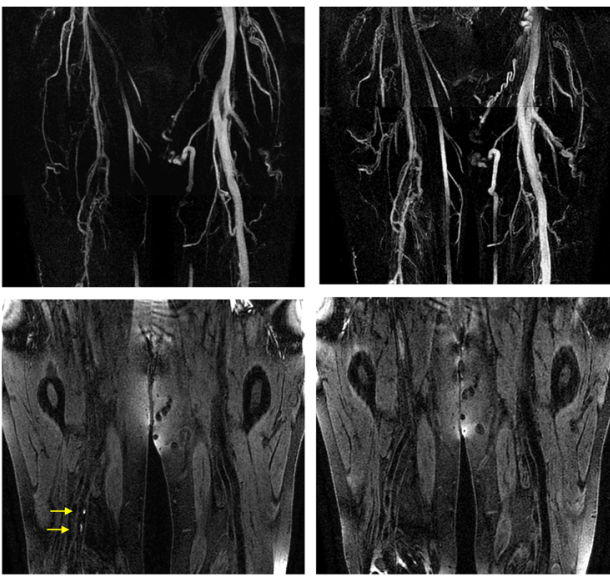

3 months

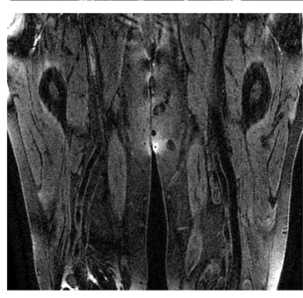

6 months unsubtracted bright- and dark-vein images, were also available to aid the diagnosis where needed.

Subsequently, an assessment of the location and length of any high-signal regions on the matching MR-DTI images was performed and these were compared with the NC-MRV images to evaluate to what extent thrombus length and location matched on both techniques. The individual-slice images were evaluated with reference to curved-reformat images, as required where the thrombi curved significantly out of the image plane. Any mismatch in thrombus extent or location was recorded. All discontinuities in high signal T1 regions were noted and the tract lengths were summed over the affected region on the matching NC-MRV.

\section{Results}

The femoral vein occlusion by thrombus was identified in all cases on NC-MRV and matched the ultrasound findings for location and extent. The thrombus length was between 6.4 and $40.0 \mathrm{~cm}$ (mean $27.7 \mathrm{~cm}$ ) on the first visit. On visit 2, thrombus was no longer present in $4 / 13$ cases, had reduced in length in a

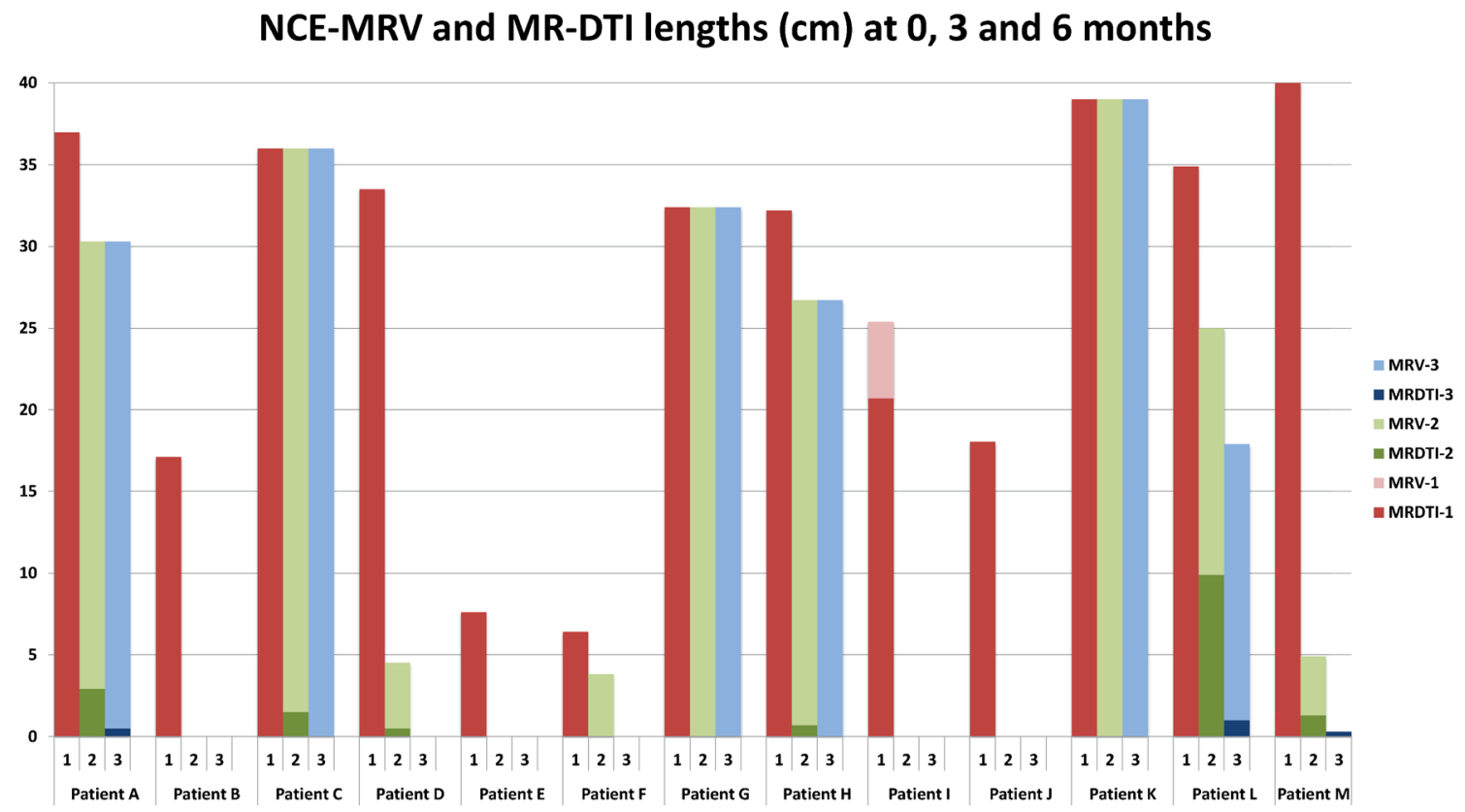

Fig. 3 Measured lengths on NC-MRV and MR-DTI images. The darker colours for each patient and visit correspond to the measurements performed on MR-DTI images (bottom bars) while the NC-MRV measurements are illustrated in lighter colours (upper bars). If, in the

same visit, the MR-DTI and NC-MRV measurements were identical, the MR-DTI value only was presented. If only NC-MRV values are shown then no measurable segment was seen on MR-DTI images 
Table 1 NC-MRV (MRV-) and MR-DTI (MRDTI-) lengths (cm) in all patients and visits

\begin{tabular}{|c|c|c|c|c|c|c|}
\hline \multirow{2}{*}{$\begin{array}{l}\text { Visit } \\
\text { Patient }\end{array}$} & \multicolumn{2}{|c|}{ Visit 1 (0 months) } & \multicolumn{2}{|c|}{ Visit 2 (3 months) } & \multicolumn{2}{|c|}{ Visit 3 (6 months) } \\
\hline & MRV-1 & MRDTI-1 & MRV-2 & MRDTI-2 & MRV-3 & MRDTI-3 \\
\hline Patient A & 37 & 37 & 30.3 & 2.9 & 30.3 & 0.5 \\
\hline Patient B & 17.1 & 17.1 & 0 & 0 & 0 & 0 \\
\hline Patient C & 36 & 36 & 36 & 1.5 & 36 & 0 \\
\hline Patient D & 33.5 & 33.5 & 4.5 & 0.5 & 0 & 0 \\
\hline Patient E & 7.6 & 7.6 & 0 & 0 & 0 & 0 \\
\hline Patient F & 6.4 & 6.4 & 3.8 & 0 & 0 & 0 \\
\hline Patient G & 32.4 & 32.4 & 32.4 & 0 & 32.4 & 0 \\
\hline Patient $\mathrm{H}$ & 32.2 & 32.2 & 26.7 & 0.7 & 26.7 & 0 \\
\hline Patient I & 25.4 & 20.7 & 0 & 0 & 0 & 0 \\
\hline Patient $\mathrm{J}$ & 18.04 & 18.04 & 0 & 0 & 0 & 0 \\
\hline Patient K & 39 & 39 & 39 & 0 & 39 & 0 \\
\hline Patient L & 34.9 & 34.9 & 25 & 9.9 & 17.9 & 1 \\
\hline Patient M & 40 & 40 & 4.9 & 1.3 & 0 & 0.3 \\
\hline
\end{tabular}

further $6 / 13$ cases (range $3.8-30.3 \mathrm{~cm}$, mean $15.9 \mathrm{~cm}$ ) and was unchanged in the remaining three cases (range 32.4-39.0 cm). On visit 3 , only $6 / 13$ cases still had thrombus present (range 17.9-39.0 cm, mean $30.4 \mathrm{~cm}$ ). Figures 1 (patient G) and 2 (patient C) show NC-MRV and MR-DTI images in two patients with venous occlusion on all three visits, while Fig. 3 shows the measured lengths of the occluded region on the NCMRV and the corresponding MR-DTI images for each visit and each patient. All thrombus length measurements are summarized in Table 1.

On the first visit, high signal at MR-DTI was found to match spatially the NC-MRV-defined occlusion in all but one case. This exception case (patient I) is shown in Fig. 4

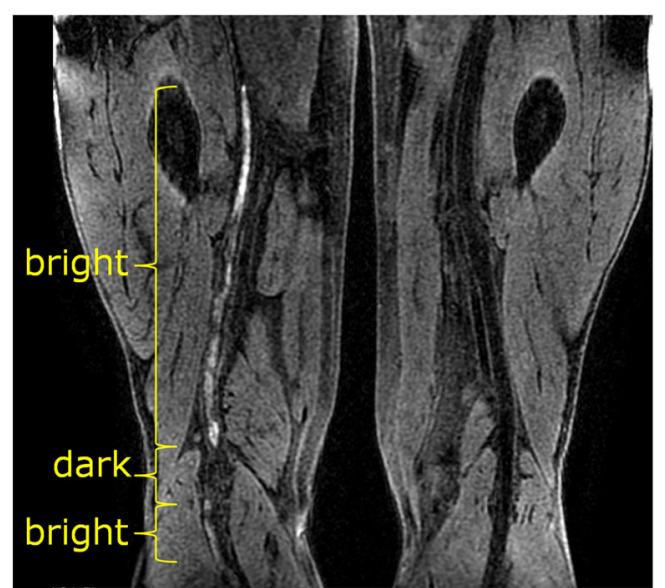

Fig. 4 MR-DTI (curved-plane reformat) of the only case where the bright-signal region on examination 1 did not fully match the occlusion observed on NC-MRV. The thrombus contained a dark-signal region near the knee with bright-signal regions both proximal and distal to it. This central dark-signal region may contain older thrombus and is discussed below. On the subsequent second and third visits, the thrombus was either not visualized or only small/ fragmented bright signal areas were seen on MR-DTI-less than $29 \%$ (visit 2) and $3 \%$ (visit 3) of the original thrombus length. The case with most residual bright thrombus signal on visits 2 and 3 is shown in Fig. 5 (patient L). Recanalisation, as assessed on the NC-MRV images, occurred in seven patients, but in six patients the superficial femoral vein remained partially or completely occluded.

\section{Discussion}

Our study is in line with previous work and confirms the potential value of MR-DTI in the diagnosis and follow-up of acute DVT involving the femoral and proximal popliteal veins by detecting the presence and changes in methaemoglobin content of the recently formed thrombus [18]. It demonstrates that by 3 months from the thrombotic event about a third of the thrombi had resolved and by 6 months only about half of the cases had residual thrombus present. When following the evolution of methaemoglobin changes over time, at 3 months less than half of the patients showed persistent, albeit small and fragmented, hyperintense areas at the site of the thrombus that almost completely cleared at the 6-month MR-DTI examination, consistent with the expected evolution of methaemoglobin.

The length and location of the thrombus matched in all patients on the NC-MRV and MR-DTI images on the first visit, except in one case (patient I shown in Fig. 4). In this patient, the proximal and distal ends of the thrombus were clearly depicted on both the NC-MRV and MR-DTI images, but the MR-DTI signal was discontinuous distally in the region of the knee. However, the extent of thrombus could still 


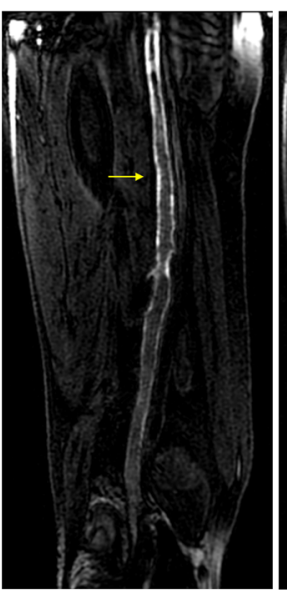

5 days

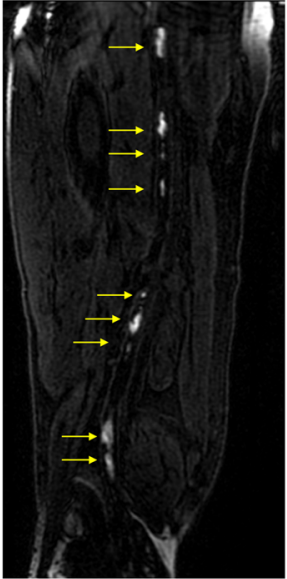

3 months

Fig. 5 MR-DTI images at all three time-points for the case showing bright-signal fragments at visit 2 ( 3 months). Transverse reformats demonstrate that these fragments are located in the centre of the thrombus, suggesting that they represent evolution of the existing

be reliably measured, as an absence of flow was demonstrated in the region of MR-DTI discontinuity.

Figure 5 (patient $\mathrm{L}$ ) shows persistent occlusion of the vein on all 3 visits with multiple short discontinuous hyperintense segments remaining at 3 months (visit 2) and reducing substantially in size at 6 months (visit 3 ). At visit 2, this exam contains the most high signal MR-DTI fragments of any examination in this study. These fragments are discontinuous and are more likely to represent residual fragmented thrombi from the initial thrombotic event, as the vein remained completely occluded (confirmed by the NC-MRV) and the bright foci were located more centrally in the vein as opposed to the peripheral "ring-like" distribution of the bright signal on the surface of the thrombus at visit 1 .

Three patients (A, L and M) had high signal intensity foci on MR-DTI images both at 3 and 6 months. In contrast to the other two patients (A and L) where these hyperintense fragments were accompanied by occlusion on NC-MRV, patient $\mathrm{M}$ showed no occlusion on the corresponding NC-MRV images. In this latter case (patient M), the bright fragments could represent either "residual" thrombus from the initial DVT or a "new" thrombus (perhaps related to an area of endothelial damage).

Our work has several strengths. We designed a longitudinal study to characterize the evolution of acute "de novo" presentations of deep vein thrombosis with two non-invasive noncontrast MR methods: a vascular technique (NC-MRV) and a thrombus-characterization technique (MR-DTI). Despite being challenging to implement because of the complexities of arterial and venous haemodynamics, we were able to perform these techniques within the constraints of a clinical workflow and acquire diagnostic quality NC-MRV and MR-DTI in a clinically acceptable examination time. We successfully

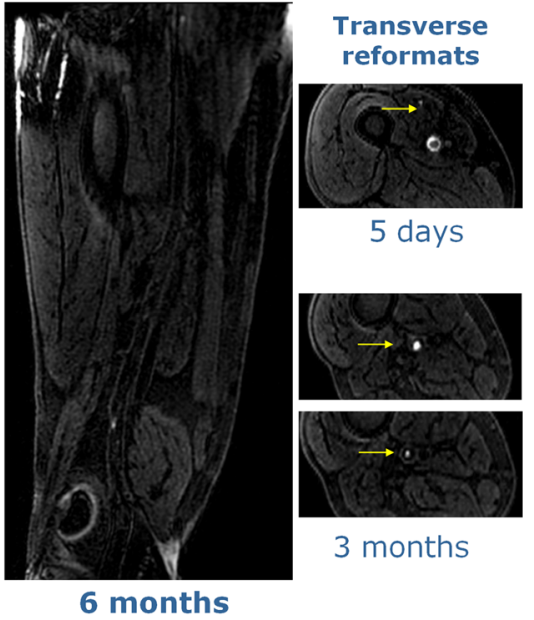

thrombus rather than development of new thrombus. In contrast, the hyperintense signal for the early time-point (visit 1) occurs around the edge of the thrombus

confirmed the expected evolution of lower-limb DVT in the majority of patients. These findings, if confirmed in a larger cohort study, could improve the diagnosis of recurrent DVT that is typically treated with lifelong anticoagulation.

The limitations of this study are the small number of patients and the use of compression US alone as the "gold standard" for thrombus detection (as invasive venogram studies are no longer performed for DVT diagnosis in our clinical practice). The recruitment criteria were designed to avoid patients with prior DVT but it is possible that an asymptomatic DVT may have occurred previously in our population as discussed regarding patient I.

Future work will concentrate on further optimization of this technique, expanding the number of patients and investigating the relationships between the observed small MR-DTI hyperintensities and the clinical evolution and risk profile of these patients.

MR-DTI, in combination with other methods such as NCMRV or US, may in future prove to be a valuable tool for distinguishing between acute and chronic thrombus in cases of suspected recurrence, potentially improving clinical management.

\section{Conclusion}

This initial study demonstrates that, in patients with a lower limb DVT, consistency between MR-DTI and NC-MRV evaluations of thrombus size and location suggests that the thrombus is acute, while a large discrepancy, or absence of high signal on MR-DTI for NC-MRV-observed thrombi, suggests an older, pre-existing thrombus. These non-invasive MRI techniques used in patients with symptoms of lower limb 
DVT might help in future avoid an incorrect diagnosis of recurrent DVT thereby avoiding the risks and complications associated with lifelong anticoagulation therapy.

Acknowledgments The authors would like to acknowledge support from the Cambridge National Institute for Health Research (NIHR) Biomedical Research Centre and Addenbrooke's Charitable Trust. The scientific guarantor of this publication is Professor DJ Lomas. This study has received funding by the NIHR Cambridge Biomedical Research Centre and Addenbrooke's Charitable Trust. No complex statistical methods were necessary for this paper. Institutional review board approval was obtained. Written informed consent was obtained from all subjects (patients) in this study. Methodology: prospective, observational, performed at one institution.

Open Access This article is distributed under the terms of the Creative Commons Attribution 4.0 International License (http:// creativecommons.org/licenses/by/4.0/), which permits unrestricted use, distribution, and reproduction in any medium, provided you give appropriate credit to the original author(s) and the source, provide a link to the Creative Commons license, and indicate if changes were made.

\section{References}

1. Michiels JJ, Michiels JM, Moossdorff W, Lao M, Maasland H, Palareti G (2015) Diagnosis of deep vein thrombosis, and prevention of deep vein thrombosis recurrence and the post-thrombotic syndrome in the primary care medicine setting anno 2014. World J Crit Care Med 4:29-39

2. Goldhaber SZ, Bounameaux H (2012) Pulmonary embolism and deep vein thrombosis. Lancet 379:1835-1846

3. Baglin T, Douketis J, Tosetto A et al (2010) Does the clinical presentation and extent of venous thrombosis predict likelihood and type of recurrence? A patient-level meta-analysis. J Thromb Haemost 8:2436-2442

4. Bates SM, Jaeschke R, Stevens SM et al (2012) Diagnosis of DVT: antithrombotic therapy and prevention of thrombosis, 9th ed: American college of chest physicians evidence-based clinical practice guidelines. Chest 141:e351S-e418S

5. Freyburger G, Trillaud H, Labrouche S et al (1998) D-dimer strategy in thrombosis exclusion-a gold standard study in 100 patients suspected of deep venous thrombosis or pulmonary embolism: 8 DD methods compared. Thromb Haemost 79:32-37

6. Perrier A, Desmarais S, Miron MJ et al (1999) Non-invasive diagnosis of venous thromboembolism in outpatients. Lancet 353:190-195

7. Tan M, van Rooden CJ, Westerbeek RE, Huisman MV (2009) Diagnostic management of clinically suspected acute deep vein thrombosis. Br J Haematol 146:347-360

8. van der Graaf F, van den Borne H, van der Kolk M, de Wild PJ, Janssen GW, van Uum SH (2000) Exclusion of deep venous thrombosis with D-dimer testing-comparison of $13 \mathrm{D}$-dimer methods in 99 outpatients suspected of deep venous thrombosis using venography as reference standard. Thromb Haemost 83:191-198

9. Michiels JJ, Gadisseur A, Van Der Planken M et al (2005) A critical appraisal of non-invasive diagnosis and exclusion of deep vein thrombosis and pulmonary embolism in outpatients with suspected deep vein thrombosis or pulmonary embolism: how many tests do we need? Int Angiol 24:27-39
10. Kearon C, Akl EA, Comerota AJ et al (2012) Antithrombotic therapy for VTE disease: antithrombotic therapy and prevention of thrombosis, 9th ed: American college of chest physicians evidence-based clinical practice guidelines. Chest 141:e419Se494S

11. Piovella F, Crippa L, Barone M et al (2002) Normalization rates of compression ultrasonography in patients with a first episode of deep vein thrombosis of the lower limbs: association with recurrence and new thrombosis. Haematologica 87:515-522

12. Mani V, Alie N, Ramachandran S et al (2015) A multicenter MRI protocol for the evaluation and quantification of deep vein thrombosis. J Vis Exp 2015:e52761

13. Linkins LA, Stretton R, Probyn L, Kearon C (2006) Interobserver agreement on ultrasound measurements of residual vein diameter, thrombus echogenicity and Doppler venous flow in patients with previous venous thrombosis. Thromb Res 117:241-247

14. Fraser DG, Moody AR, Morgan PS, Martel AL, Davidson I (2002) Diagnosis of lower-limb deep venous thrombosis: a prospective blinded study of magnetic resonance direct thrombus imaging. Ann Intern Med 136:89-98

15. Saha P, Andia ME, Modarai B et al (2013) Magnetic resonance T1 relaxation time of venous thrombus is determined by iron processing and predicts susceptibility to lysis. Circulation 128:729-736

16. Moody AR (2003) Magnetic resonance direct thrombus imaging. J Thromb Haemost 1:1403-1409

17. Moody AR, Pollock JG, O'Connor AR, Bagnall M (1998) Lowerlimb deep venous thrombosis: direct MR imaging of the thrombus. Radiology 209:349-355

18. Tan M, Mol GC, van Rooden CJ et al (2014) Magnetic resonance direct thrombus imaging differentiates acute recurrent ipsilateral deep vein thrombosis from residual thrombosis. Blood 124:623-627

19. Westerbeek RE, Van Rooden CJ, Tan M et al (2008) Magnetic resonance direct thrombus imaging of the evolution of acute deep vein thrombosis of the leg. J Thromb Haemost 6:1087-1092

20. Mani V, Alie N, Ramachandran S et al (2015) A multicenter MRI protocol for the evaluation and quantification of deep vein thrombosis. J Vis Exp. doi:10.3791/52761

21. Ono A, Murase K, Taniguchi T et al (2010) Deep venous thrombosis: diagnostic value of non-contrast-enhanced MR venography using electrocardiography-triggered three-dimensional halfFourier FSE. Magn Reson Med 64:88-97

22. Priest AN, Graves MJ, Lomas DJ (2012) Non-contrast-enhanced vein imaging in the deep veins: impact of velocity patterns and improved image quality. Proc Intl Soc Magn Reson Med 20:1210

23. Priest AN, Joubert I, Hilborne S, Hunter S, Bowden DJ, Graves MJ, Baglin T, Lomas DJ (2013) Non-contrast-enhanced imaging of lower limb veins: improved imaging using multiple flow preparations. Proc Intl Soc Magn Reson Med 21:1287

24. Priest AN, Graves MJ, Lomas DJ (2012) Non-contrast-enhanced vascular magnetic resonance imaging using flow-dependent preparation with subtraction. Magn Reson Med 67:628-637

25. Priest AN, Taviani V, Graves MJ, Lomas DJ (2014) Improved artery-vein separation with acceleration-dependent preparation for non-contrast-enhanced magnetic resonance angiography. Magn Reson Med 72:699-706

26. Priest AN, Joubert I, Hilborne S, Hunter S, Bowden DJ, Graves MJ, Baglin T, Gillard JH, Lomas DJ (2013) MR direct thrombus imaging with optimised signal and improved lipid suppression. Proc Intl Soc Magn Reson Med 21:1280 